\title{
Acute Stress-Induced Coagulation Activation in Patients With Remitted Major Depression Versus Healthy Controls and the Role of Stress-Specific Coping
}

von Känel, Roland ; Merz, Franziska ; Pfister, Hildegard ; Brückl, Tanja ; Zimmermann, Petra ; Uhr, Manfred ; Holsboer, Florian ; Höhne, Nina ; Ising, Marcus

\begin{abstract}
Background: Depressed patients have an increased risk of myocardial infarction, for which acute stress is a frequent trigger. Prothrombotic changes could be one involved mechanism that can be modulated by psychological coping. Purpose: We examined the effects of remitted major depression and situation-specific coping strategies on stress-induced coagulation activation. Methods: Forty patients with remitted depression and 23 healthy controls underwent the Trier Social Stress Test, rating applied coping strategies thereafter. Blood was sampled at baseline and 15 and 45 min poststress to measure fibrinogen, von Willebrand factor (VWF) and D-dimer. Coagulation activation over time was quantified as area under the curve (AUC) with respect to baseline activity. Standardized z-scores of individual coagulation AUC measures were added up to a prothrombotic index. Results: Stress provoked significant VWF (p $=.024)$ and D-dimer $(\mathrm{p}=.002)$ responses. Remitted depressed patients used positive distraction coping more frequently than controls did $(\mathrm{p}=.030)$. Coagulation AUC measures were similar in both groups. In all participants, higher positive coping total $(\mathrm{p}=0.009)$, driven by devaluation/defense $(\mathrm{p}=.022)$ and distraction $(\mathrm{p}=.004)$ coping, was associated with a lower prothrombotic index. In controls, but not in remitted depressed patients, higher positive coping total $(\mathrm{p}=.008)$, driven by higher devaluation/defense $(\mathrm{p}=.010)$ and distraction $(\mathrm{p}=.023)$ coping, was associated with lower VWF AUC. Conclusions: Despite the use of favorable coping strategies in a specific stress situation, remitted depressed patients may benefit less from a positive effect of positive situational coping on coagulation activation than controls. Such a mechanism could partially explain the increased risk of myocardial infarction in depressed individuals.
\end{abstract}

DOI: https://doi.org/10.1093/abm/kaaa001

Posted at the Zurich Open Repository and Archive, University of Zurich ZORA URL: https://doi.org/10.5167/uzh-198817

Journal Article

Accepted Version

Originally published at:

von Känel, Roland; Merz, Franziska; Pfister, Hildegard; Brückl, Tanja; Zimmermann, Petra; Uhr, Manfred; Holsboer, Florian; Höhne, Nina; Ising, Marcus (2020). Acute Stress-Induced Coagulation Activation in Patients With Remitted Major Depression Versus Healthy Controls and the Role of Stress-Specific Coping. Annals of Behavioral Medicine, 54:611-618.

DOI: https://doi.org/10.1093/abm/kaaa001 


\section{Acute stress-induced coagulation activation in patients with remitted major depression} versus healthy controls and the role of stress-specific coping

Short title: Prothrombotic reactivity, depression and coping

${ }^{1}$ Roland von Känel, MD *; ${ }^{2}$ Franziska Merz, MD *; ${ }^{2}$ Hildegard Pfister, MSc;

${ }^{2}$ Tanja Brückl, PhD; ${ }^{2}$ Petra Zimmermann, $\mathrm{PhD} ;{ }^{2}$ Manfred Uhr, MD, PhD;

${ }^{2,3}$ Florian Holsboer, MD, PhD; ${ }^{2,4}$ Nina Höhne, $\mathrm{PhD} * * ;{ }^{2}$ Marcus Ising, $\mathrm{PhD} * *$

${ }^{1}$ Department of Consultation-Liaison Psychiatry and Psychosomatic Medicine, University Hospital Zurich, University of Zurich, Zurich, Switzerland

${ }^{2}$ Max Planck Institute of Psychiatry, Munich, Germany

${ }^{3}$ HMNC Brain Health GmbH, Munich, Germany

${ }^{4}$ Centre for Digitization Bavaria, Munich, Germany

*,** These authors contributed equally to this work.

\section{Address for correspondence:}

Roland von Känel, MD

Department of Consultation-Liaison Psychiatry and Psychosomatic Medicine University Hospital Zurich

Culmannstrasse 8

CH-8091 Zurich / Switzerland

Tel.: +41 (0)44 25552 51; Fax: +41 (0)44 25544 08; E-mail: roland.vonkaenel@ usz.ch 


\begin{abstract}
Background: Depressed patients have an increased risk of myocardial infarction, for which acute stress is a frequent trigger. Prothrombotic changes are one involved mechanism, which can be modulated by psychological coping.
\end{abstract}

Purpose: We examined the effects of remitted major depression and situation-specific coping strategies on stress-induced coagulation activation.

Methods: 40 remitted depressed patients and 23 healthy controls underwent the Trier Social Stress Test, rating applied coping strategies thereafter. Blood was sampled at baseline, $15 \mathrm{~min}$ and $45 \mathrm{~min}$ post-stress to measure fibrinogen, von Willebrand factor (VWF) and D-dimer. Coagulation activation over time was quantified as area under the curve (AUC) with respect to baseline activity. Standardized z-scores of individual coagulation AUC measures were added up to a prothrombotic index.

Results: Stress provoked significant VWF ( $\mathrm{p}=0.024)$ and D-dimer $(\mathrm{p}=0.002)$ responses. Remitted depressed patients used positive distraction coping more frequently than controls did $(\mathrm{p}=0.030)$. Coagulation AUC measures were similar in both groups. In all participants, higher positive coping total $(p=0.009)$, driven by devaluation/defense $(p=0.022)$ and distraction $(p=0.004)$ coping, was associated with a lower prothrombotic index. In controls, but not in remitted depressed patients, higher positive coping total $(\mathrm{p}=0.008)$, driven by higher devaluation/defense $(\mathrm{p}=0.010)$ and distraction $(\mathrm{p}=0.023)$ coping, was associated with lower VWF AUC.

Conclusions: Despite the use of favorable coping strategies in a specific stress situation, remitted depressed patients may benefit less from a positive effect of positive situational coping on coagulation activation than controls. Such a mechanism could partially explain the increased risk of myocardial infarction in depressed individuals.

Keywords: Biomarker, blood coagulation, cardiovascular disease, coping, depression, stress 


\section{INTRODUCTION}

Major depressive disorder (MDD) is associated with a 1.3-fold increased risk of incident myocardial infarction (MI) and coronary death (1). Minor symptoms of depression have also been associated with an excess risk of MI, independent of sociodemographic and traditional cardiovascular risk factors $(2,3)$. Between $10 \%$ and $20 \%$ of patients report an emotional trigger within 1-2 hours of MI onset $(4,5)$, with, for instance, acute depressed mood quadrupling the risk (6). Individuals with subclinical atherosclerosis, prevalent in depression, are particularly vulnerable to mental triggering of MI $(7,8)$. Several biological mechanisms are involved in mental triggering of MI. Activation of the sympathetic nervous system (SNS) results in increased myocardial oxygen demand, vasoconstriction, hemodynamic shear forces to a vulnerable atherosclerotic plaque, and proinflammatory and procoagulant changes $(9,10)$. Regarding the latter, thrombotic occlusion following plaque rupture marks the transition from subclinical atherosclerosis to acute MI (11). This is especially true when excessive coagulation activation occurs beyond a physiologic procoagulant response to stress, the purpose of which is to protect a healthy organism from too much blood loss in fight-flight situations (10).

Depression has been shown to be a modulator of stress-induced coagulation activation and post-stress recovery (10). For instance, in older dementia caregivers, depressed mood was associated with increased stress-induced platelet activation and delayed recovery of platelet activity post-stress (12). In middle-aged adults, depressed mood was also associated with a stress-induced increase in plasma levels of von Willebrand factor (VWF), mediating platelet adhesion and aggregation (13), and with delayed post-stress recovery in D-dimer levels, indicating prolonged intravascular fibrin formation (14). One study found increased stress reactivity of platelets in patients meeting clinical criteria for current MDD (15). As yet, there are no studies on stress-induced coagulation activation in patients with remitted MDD. 
This knowledge could reveal whether the procoagulant stress response is a state or trait marker of MDD and may have implications for cardiovascular disease and prevention.

Positive, adaptive coping strategies may attenuate the intensity and duration of the acute procoagulant stress response, whereas negative, maladaptive strategies seem less influential. In older dementia caregivers, greater levels of approach coping and planful problem-solving were both associated with lower D-dimer levels following a speech challenge and during post-stress recovery, whereas avoidant coping showed no relation with D-dimer levels (16). Healthy male subjects with greater adaptive anticipatory cognitive stress appraisal (e.g., less threat perception) showed less D-dimer formation in response to the Trier Social Stress Test (TSST) and during a post-stress recovery interval of one hour (17). When faced with general stress conditions, depressed individuals report less positive dispositional (trait) coping strategies, although more negative ones, than their non-depressed counterparts $(18,19)$. Whether situational (state) positive and negative coping strategies differ between depressed individuals and controls is to our knowledge unknown. However, as MI is being triggered by acute stress situations, studying the effects of stress-specific coping strategies on coagulation reactivity, as opposed to general ones, could yield deeper pathophysiologic insight.

Our primary aim was to examine whether remitted MDD patients differ from healthy controls in mental stress-induced activation and post-stress recovery in fibrinogen, VWF, and D-dimer levels, and a sum score of these coagulation measures (prothrombotic index, PTI). Our secondary aim was to test the hypothesis that greater use of state positive coping is associated with less coagulation activation and to explore whether remitted MDD patients would differ from controls regarding such a stress-buffering effect. We also tested the tentative assumption (16) that negative state coping is less influential in affecting procoagulant stress responses than positive state coping. 


\section{MATERIALS AND METHODS}

\section{Study participants and recruitment}

This study was embedded in the second of two recruitment waves of a larger project investigating gene-environment effects on the laboratory stress response in remitted MDD (20). Results of the first recruitment wave have been reported elsewhere (19). Patients with remitted MDD and healthy controls were recruited from the longitudinal Early Developmental Stages of Psychopathology (EDSP) study, originally designed to investigate the prevalence, incidence, risk factors, course and comorbidity of mental disorders. In 1994, a random sample of 3,021 individuals aged 14 to 24 years of the city of Munich's population register underwent the Munich-Composite International Diagnostic Interviews (M-CIDI) for the identification of DSM-IV psychiatric disorders (21). Four interviews were performed between 1995 and 2005 with 2,210 subjects having participated in the final interview (22). We used the aggregated diagnostic information across all EDPS assessments resulting in 403 eligible subjects fulfilling the inclusion criteria as described below. From these subjects, 299 could be reached by telephone and were invited to participate in the laboratory stress test study. 139 refused participation for various reasons and further 29 subjects were excluded for medical reasons (severe medical disorder including ischemic heart disorder, cancer, endocrine disorders, medication with glucocorticoids, report of acute depressive symptoms). 131 subjects finally participated in the stress test study, with 66 recruited in the second recruitment wave. Coagulation activation as presented in here was measured only in the 66 participants of the second recruitment wave.

Inclusion criteria for the remitted MDD patient group were a lifetime diagnosis of MDD and full remission of a MDD episode for at least six months. Lifetime diagnoses of a psychotic disorder, substance-abuse disorder, social phobia, bipolar affective disorder, dysthymia, needle-related anxiety, and medical conditions affecting coagulation activity were 
exclusion criteria for all participants, but antidepressant use was not. For the healthy control group lifetime diagnoses of affective disorders and generalized anxiety disorder were additional exclusion criteria. The study personnel was kept blinded regarding the diagnostic information of the participants to ensure unbiased recruitment and study procedure for both groups. The ethics committee of the Ludwig-Maximilian University, Munich, Germany, approved the study protocol. All participants provided consent.

\section{Diagnostic assessment and measures}

Information on medication use, demographics, smoking history, and weight and height to calculate the body mass index (BMI) was obtained. All participants completed the German version of the 21-item Beck Depression Inventory (BDI)-II (23). Out of 66 recruited participants three had to be excluded, two because of depressive symptoms (BDI-II score $\geq 14$; one from each group), and one healthy control with suspected acute infection based on a Creactive protein $(\mathrm{CRP})$ level of $28.7 \mathrm{mg} / \mathrm{L}$ influencing coagulation. Hence, 63 participants, 40 with remitted MDD and 23 controls were available for analysis. Further details on the study procedure have been published elsewhere $(19,20)$.

\section{Experimental procedure}

After arrival at the laboratory at 1:15 pm, participants were equipped with a pulse watch and chest belt (Polar@RS400) to measure heart rate (HR) as an index of stress-induced SNS activation. A venous catheter was placed into the vein of the non-dominant forearm 30 min prior to collection of the baseline blood sample. To induce stress, we applied the widely used TSST (24) with an instruction and preparation phase of 10 minutes each, followed by a 10-min public speaking task, involving a mock job interview and mental arithmetic. Participants watched a movie during a 45-min stress recovery phase. Blood samples for 
coagulation measures were taken at baseline, and $15 \mathrm{~min}$ and $45 \mathrm{~min}$ post-stress. The TSST has been shown to provoke significant increases in plasma levels of coagulation factors, including fibrinogen, VWF, and D-dimer $(14,17,25)$.

About 30 min into the recovery phase from the TSST, all participants completed the 84-item version of the German Stress Coping Inventory ("Stressverarbeitungsfragebogen", SVF84-ak) (26). The questionnaire allows a retrospective self-assessment of coping strategies which were being used to deal with the preceding stressful situation. Participants were asked how likely coping-related thoughts came to their mind during the TSST such as "I try to control/antagonize my arousal". Each item is rated on a Likert-scale from 0 ("not at all") to 3 ("certainly"). Four of the 84 items are summed up into 21 subscales, each, with missing items (less than $0.1 \%$ ) replaced by the respective mean of the remaining items of a subscale. According to factor analysis, subscales can be clustered into one category with negative situational coping strategies, and one category with positive situational coping strategies. Negative coping comprises the subscales escape, social withdrawal, rumination, resignation, helplessness, self-pity, and self-blame (Cronbach alpha $(\alpha)=.92)$. Positive coping total $(\alpha=.90)$ can further be categorized into three categories termed (subscales in parentheses) 1) devaluation/defense (minimization, self-aggrandizement by comparison with others, denial of guilt; $\alpha=80$ ), 2) distraction (distraction, substitute gratification, search for self-affirmation, relaxation; $\alpha=.84$ ), and 3 ) control (situation control, reaction control, positive selfinstructions; $\alpha=.85$ ). Each category is calculated as the mean of the respective subscale scores. The total possible range of all subscale and category scores is between 0 and 12, each.

\section{Biochemical analyses}

Venous blood was collected into $0.3 \mathrm{~mL}$ citrate buffer tubes (Monovettes, Sarstedt, Nuremberg, Germany). Plasma concentrations of fibrinogen (lower limit of detection, LOD: 
$30 \mathrm{mg} / \mathrm{dL}$ ) and VWF antigen (lower LOD: $270 \mathrm{mg} / \mathrm{L}$ ) were determined with a multiplexed particle-based flow cytometric assay (Cytolab, Regensdorf, Switzerland). Plasma D-dimer concentration (lower LOD: $0 \mathrm{ng} / \mathrm{mL}$ ) was determined with an enzyme-linked immunosorbent assay (Technozym D-dimer, Technoclone, Vienna, Austria). Inter- and intra-assay coefficients of variation were $<15 \%$ for all analyses. CRP plasma levels were determined with the cobas 6000 system (Roche Diagnostics, Switzerland).

\section{Data analysis}

Data were analyzed using SPSS statistics 25 (SPSS Inc., Chicago IL, USA) with significance level of $\mathrm{p}<0.05$ (two-tailed). Effect sizes are expressed as Cohen's d where 0.2, 0.5, and 0.8 reflect small, medium and large effects, respectively. Missing values for BDI-IIscores $(n=2)$, fibrinogen $(n=2), \operatorname{VWF}(n=5)$ and D-dimer $(n=8)$ were replaced using the expectation maximization algorithm. D-dimer and CRP values were log transformed. Group differences in continuous and categorical data were calculated using independent samples ttest and chi-square test, respectively. Bivariate relationships were estimated with Pearson correlation coefficients. Stress responses in coagulation measures and HR were analyzed using repeated measures analysis of variance with the Greenhouse-Geisser correction of degrees of freedom. Changes in biological variables over time were quantified (arbitrary units) with the area under the curve (AUC) with respect to baseline values (27). To calculate the PTI, our primary outcome variable, standardized z-scores of AUC values of fibrinogen, VWF and $\log$ D-dimer were added up, and the total was divided by three. In accordance with a systems biology approach (28), the PTI reflects an integrated estimate of prothrombotic function. Linear regression analysis, using forced entry, was employed to identify whether "group" (remitted MDD vs. healthy controls), negative and positive coping strategies, and the interaction terms between group and coping strategies were significantly linked with AUC of 
coagulation measures. To guard against model overfitting, a maximum of four covariates (i.e., age, sex, BMI, current smoking) were selected a priori which could affect coagulation activity. Additional covariates were separately tested for potential influences. A sensitivity analysis was run excluding participants using a selective serotonin reuptake inhibitor (SSRI) known to influence coagulation.

\section{RESULTS}

\section{Participant characteristics}

As shown in Table 1, health characteristics were similar in both groups, except of a higher distraction score in patients with remitted MDD. Average levels of depressive symptoms were in the non-clinical range in both groups. Four participants took antidepressants, all an SSRI.

\section{Biological responses to acute stress}

Repeated measure ANOVA showed significant changes in $\mathrm{HR}(\mathrm{F}=150, \mathrm{p}<0.001$, $\mathrm{d}=2.81)$ and in plasma levels of $\operatorname{VWF}(\mathrm{F}=4.47, \mathrm{p}=0.024, \mathrm{~d}=0.54)$ and $\mathrm{D}$-dimer $(\mathrm{F}=7.53$, $\mathrm{p}=0.002, \mathrm{~d}=0.70)$, but not in fibrinogen $(\mathrm{p}=0.20)$ across all participants. VWF and D-dimer levels were higher than baseline $15 \mathrm{~min}$ and $45 \mathrm{~min}$ post-stress (all p-values $\leq 0.42$ ). There were no significant group-by-time interactions for HR $(p=0.19)$, fibrinogen $(p=0.60)$, VWF $(\mathrm{p}=0.76)$ and $\mathrm{D}$-dimer $(\mathrm{p}=0.25)$. In Table 2 , mean values for HR and coagulation factors across time points are depicted for each group.

\section{Remitted depression, coping and stress-induced coagulation activation over time}

As can be seen in Table 3, unlike positive coping total, negative coping showed no significant associations with coagulation measures in the unadjusted analyses (Model 1b), the 
analyses adjusted for age, sex, BMI, and smoking (Model 2a), and in the adjusted moderator analyses (Model 3a). The non-significant results for negative coping were confirmed in a sensitivity analysis, excluding the four participants with SSRI use.

Higher positive coping total was associated with lower change in PTI AUC in the adjusted analysis ( $\mathrm{p}=0.009, \mathrm{~d}=0.73$; Model $2 \mathrm{~b}$ ) in all participants. A significant group-bypositive coping total interaction emerged for VWF AUC ( $\mathrm{p}=0.002, \mathrm{~d}=0.87$, Model $3 \mathrm{~b})$ such that higher positive coping total was significantly associated with lower VWF AUC in healthy controls $(B=-0.494,95 \%$ CI: $-0.842,-0.146, p=0.008, d=1.45)$, but not in remitted MDD patients $(B=-0.018,95 \% \mathrm{CI}:-0.179,0.144, \mathrm{p}=0.83, \mathrm{~d}=0.08)$. In the latter, additional adjustment for time since the last depressive episode did not change this association $(\mathrm{p}=0.97)$. In a sensitivity analysis, excluding participants using an SSRI, the significance of the relationship between positive coping total and PTI AUC $(\mathrm{p}=0.019)$ and of the group-bypositive coping total interaction for VWF AUC ( $\mathrm{p}=0.18)$ were both maintained. Further complementary analyses were performed to examine potentially confounding effects of additional variables. The significance of the above results for positive coping total was maintained when either negative coping, HR AUC, oral contraceptive use, CRP levels, residual depressive symptoms or the number of previous MDD episodes were additionally adjusted for, each separately so to limit the risk of model overfitting.

\section{Associations with subscales of positive coping}

To specify the characteristics of positive coping being associated with the above reported stress-induced coagulation activation, we modelled separate analyses for each positive coping subscale. All subscales showed an inverse association, reaching significance for devaluation/defense and distraction, with PTI AUC in all participants and with VWF AUC in healthy controls relative to patients with remitted MDD (Table 4). 


\section{DISCUSSION}

This study examined acute stress-induced coagulation activation along with a potentially modulating role of stress-specific coping strategies in patients with remitted MDD and healthy controls. Across all participants, corroborating previous studies (13, 14, 16, 17 , 25), plasma levels of VWF and D-dimer, although not fibrinogen, showed a significant stress response, remaining higher than at baseline during a post-stress recovery interval of $45 \mathrm{~min}$. This is within the critical time interval during which the risk of MI onset increases following emotional upset (29). However, coagulation responses over time were not significantly different between patients with remitted MDD and healthy controls, even when considering potentially confounding effects of a range of variables that could affect coagulation. This observation contrasts with several previous studies showing significant relationships between current depressed mood severity and stress-induced coagulation changes (12-15). Therefore, it is arguably the chronic trait factor of current depression which might critically underlie prothrombotic stress reactivity.

Across all study participants, we found that greater levels of situational positive coping total, significantly driven by positive devaluation/defense and distraction coping, was associated with less pronounced coagulation activation over time measured by a PTI. The same significant associations were found for lower changes in VWF over time in healthy controls, but not in remitted MDD patients. All associations showed moderate to large effect sizes, suggesting that the absence of positive situational coping strategies could have clinical implications such as for coagulation mechanisms involved in stress-triggered MI. By and large, this novel finding is consistent with the general view that positive dispositional coping strategies are adaptive, alleviate stress, and thus provide health benefits. In this regard, healthy controls seemed to benefit more from positive state coping effects on VWF reactivity than patients with remitted MDD. This finding is particularly intriguing given that remitted 
MDD patients reported significantly greater use of positive distraction coping in dealing with the TSST than controls. Impaired endothelial function in patients with remitted MDD (30) could be one explanation for a group difference in VWF reactivity, but not in fibrinogen and D-dimer reactivity. Unlike fibrinogen and D-dimer, VWF is released from endothelial cells into circulation in response to stress and impaired endothelial function is associated with excess VWF release (10). However, the mechanisms and implications of this group difference for cardiovascular health outcomes were not examined and therefore remain speculative. Similar to a previous study (16), stress-specific negative coping was not associated with coagulation reactivity.

Besides the lack of a comparison group with current MDD, the possibility of residual confounding due to unmeasured lifestyle and subclinical atherosclerosis, lacking information on psychotherapy treatment, and limited statistical power, the interpretation of our findings should consider several limitations. We measured coagulation factors 15 min into the post-stress recovery period, instead of immediately after stress, which could help to explain the lack of both a stress response in fibrinogen and an association of positive coping with fibrinogen and D-dimer. In contrast, VWF is arguably among the most responsive hemostatic factors to sympathetic activation and stress (10). Although depressed individuals exhibit greater moment-to-moment variability and daily life-event-related stress reactivity in negative affect $(31,32)$, it is as yet unknown whether they also run a higher risk of incident MI with emotional triggers than their non-depressed counterparts. Whether biological responses like coagulation changes to the TSST can be translated to real life stress situations is a matter of debate. Additional assessment of stress responses in platelets, clotting factor VIII and fibrinolysis had yielded a more comprehensive picture of hemostatic changes to stress (10).

Taken together, remitted MDD patients did not differ in stress-induced coagulation responses from healthy controls. Positive stress-specific coping strategies may mitigate stressinduced coagulation activation, and this effect seemed less pronounced in patients with remitted 
MDD regarding VWF reactivity. Implications for the prevention of stress-triggered MI could comprise adopting positive state coping in patients at risk of cardiovascular disease, including those with a lifetime history of MDD.

\section{REFERENCES}

1. Wu Q, Kling JM. Depression and the Risk of Myocardial Infarction and Coronary Death: A Meta-Analysis of Prospective Cohort Studies. Medicine (Baltimore). 2016; 95(6): e2815.

2. Gan Y, Gong Y, Tong X, et al. Depression and the risk of coronary heart disease: a metaanalysis of prospective cohort studies. BMC Psychiatry. 2014; 14:3 71.

3. Van der Kooy K, van Hout H, Marwijk H, Marten H, Stehouwer C, Beekman A. Depression and the risk for cardiovascular diseases: systematic review and meta analysis. Int J Geriatr Psychiatry. 2007; 22: 613-626.

4. Tofler GH, Kopel E, Klempfner R, Eldar M, Buckley T, Goldenberg I; National Israel Survey of Acute Coronary Syndrome Investigators. Triggers and Timing of Acute Coronary Syndromes. Am J Cardiol. 2017; 119: 1560-1565.

5. Tofler GH, Stone PH, Maclure M, et al. Analysis of possible triggers of acute myocardial infarction (the MILIS study). Am J Cardiol. 1990; 66: 22-27.

6. Steptoe A, Strike PC, Perkins-Porras L, McEwan JR, Whitehead DL. Acute depressed mood as a trigger of acute coronary syndromes. Biol Psychiatry. 2006; 60:837-842.

7. Leor J, Poole WK, Kloner RA. Sudden cardiac death triggered by an earthquake. N Engl J Med. 1996; 334: 413-419.

8. Wu Y, Sun D, Wang B, Li Y, Ma Y. The relationship of depressive symptoms and functional and structural markers of subclinical atherosclerosis: A systematic review and meta-analysis. Eur J Prev Cardiol. 2018; 25: 706-716. 
9. Edmondson D, Newman JD, Whang W, Davidson KW. Emotional triggers in myocardial infarction: do they matter? Eur Heart J. 2013; 34: 300-306.

10. Austin AW, Wissmann T, von Kanel R. Stress and hemostasis: an update. Semin Thromb Hemost. 2013; 39: 902-912.

11. Abbate R, Cioni G, Ricci I, Miranda M, Gori AM. Thrombosis and acute coronary syndrome. Thromb Res. 2012; 129: 235-240.

12. Aschbacher K, Mills PJ, von Känel R, et al. Effects of depressive and anxious symptoms on norepinephrine and platelet P-selectin responses to acute psychological stress among elderly caregivers. Brain Behav Immun. 2008; 22: 493-502.

13. von Känel R, Hamer M, Malan NT, Scheepers K, Meiring M, Malan L. Procoagulant reactivity to laboratory acute mental stress in Africans and Caucasians, and its relation to depressive symptoms: the SABPA study. Thromb Haemost. 2013; 110: 977-986.

14. von Känel R, Bellingrath S, Kudielka BM. Association of vital exhaustion and depressive symptoms with changes in fibrin D-dimer to acute psychosocial stress. J Psychosom Res. 2009; 67: 93-101.

15. Plourde A, Lavoie KL, Ouellet K, Carroll D, Ring C, Bacon SL. Hemodynamic, hemostatic, and endothelial reactions to acute psychological stress in depressed patients following coronary angiography. Psychophysiology. 2013; 50: 790-798.

16. Aschbacher K, Patterson TL, von Känel R, et al. Coping processes and hemostatic reactivity to acute stress in dementia caregivers. Psychosom Med. 2005; 67: 964-971.

17. Wirtz PH, Ehlert U, Emini L, et al. Anticipatory cognitive stress appraisal and the acute procoagulant stress response in men. Psychosom Med. 2006; 68: 851-858.

18. Sun XJ, Niu GF, You ZQ, Zhou ZK, Tang Y. Gender, negative life events and coping on different stages of depression severity: A cross-sectional study among Chinese university students. J Affect Disord. 2017; 209: 177-181. 
19. Höhne N, Poidinger M, Merz F, et al. Increased HPA axis response to psychosocial stress in remitted depression: the influence of coping style. Biol Psychol. 2014; 103: 267-275.

20. Höhne N, Poidinger M, Merz F, et al. FKBP5 genotype-dependent DNA methylation and mRNA regulation after psychosocial stress in remitted depression and healthy controls. Int J Neuropsychopharmacol. 2015; 18: 1-9.

21. Wittchen HU, Pfister H. DIA-X-Interviews: Manual für Screening-Verfahren und Interview. Interviewheft Längsschnittuntersuchung (DIA-X lifetime); Ergänzungsheft (DIA-X); Interviewheft Querschnittsuntersuchung (DIA-X 12 Monate); Ergänzungsheft (DIA-X 12 Monate); Frankfurt: Swets \& Zeitlinger, 1997.

22. Beesdo-Baum K, Knappe S, Asselmann E, et al. The 'Early Developmental Stages of Psychopathology (EDSP) study': a 20-year review of methods and findings. Soc Psychiatry Psychiatr Epidemiol. 2015; 50:851-866.

23. Hautzinger M, Keller F, Kühner C. BDI-II-Beck Depressions-Inventar Revision. Frankfurt/Main: Pearson, 2006.

24. Kirschbaum C, Pirke KM, Hellhammer DH. The 'Trier Social Stress Test'--a tool for investigating psychobiological stress responses in a laboratory setting. Neuropsychobiology. 1993; 28: 76-81.

25. von Känel R, Preckel D, Zgraggen L, et al. The effect of natural habituation on coagulation responses to acute mental stress and recovery in men. Thromb Haemost. 2004; 92: 1327-1335.

26. Erdmann G, Janke W. Stressverarbeitungsfragebogen (SVF). Stress, Stressverarbeitung und ihre Erfassung durch ein mehrdimensionales Testsystem. Göttingen: Hogrefe, 2008.

27. Pruessner JC, Kirschbaum C, Meinlschmid G, Hellhammer DH. Two formulas for computation of the area under the curve represent measures of total hormone 
concentration versus time-dependent change. Psychoneuroendocrinology. 2003; 28: 916931.

28. Diamond SL. Systems biology of coagulation. J Thromb Haemost 2013; 11 Suppl 1: 224232.

29. Mostofsky E, Penner EA, Mittleman MA. Outbursts of anger as a trigger of acute cardiovascular events: a systematic review and meta-analysis. Eur Heart J. 2014; 35: 1404-1410.

30. Broadley AJ, Korszun A, Jones CJ, Frenneaux MP. Arterial endothelial function is impaired in treated depression. Heart. 2002; 8: 521-523.

31. Peeters F, Berkhof J, Delespaul P, Rottenberg J, Nicolson NA. Diurnal mood variation in major depressive disorder. Emotion. 2006; 6: 383-391.

32. Wichers M, Myin-Germeys I, Jacobs N, et al. Genetic risk of depression and stressinduced negative affect in daily life. Br J Psychiatry. 2007; 191: 218-223. 
Table 1. Characteristics of the 63 study participants

\begin{tabular}{|c|c|c|c|}
\hline Variables & $\begin{array}{l}\text { Remitted MDD } \\
(\mathrm{n}=40)\end{array}$ & $\begin{array}{l}\text { Controls } \\
(n=23)\end{array}$ & $\mathrm{P}$ \\
\hline Age, years & $34.7(3.5)$ & $36.1(3.6)$ & 0.128 \\
\hline Female sex, $\mathrm{n}$ & $23(57.5)$ & $14(65.2)$ & 0.794 \\
\hline Oral contraceptives, $\mathrm{n}$ & $7(17.5)$ & $6(26.1)$ & 0.522 \\
\hline Selective serotonin reuptake inhibitors, $\mathrm{n}$ & $2(5.0)$ & $2(8.7)$ & 0.465 \\
\hline Body mass index, $\mathrm{kg} / \mathrm{m}^{2}$ & $23.9(2.9)$ & $23.7(2.4)$ & 0.797 \\
\hline Current smoker, $\mathrm{n}$ & $8(20.0)$ & $5(21.7)$ & 1.000 \\
\hline $\mathrm{C}$-reactive protein, $\mathrm{mg} / \mathrm{L}$ & $2.24(2.48)$ & $1.98(1.98)$ & 0.728 \\
\hline Age of depression onset, years & $19.2(4.4)$ & n.a. & n.a. \\
\hline Time since last depressive episode, years & $10.6(6.3)$ & n.a. & n.a. \\
\hline Previous depressive episodes, $\mathrm{n}$ & $3.3(3.3)$ & 0 & $<0.001$ \\
\hline BDI-II symptoms, score & $2.0(2.8)$ & $2.0(3.3)$ & 0.968 \\
\hline Negative coping, score & $1.4(1.6)$ & $1.2(1.3)$ & 0.659 \\
\hline Positive coping total, score & $3.6(1.8)$ & $3.5(1.7)$ & 0.676 \\
\hline Positive coping devaluation/defense, score & $3.0(1.6)$ & $3.0(2.0)$ & 0.985 \\
\hline Positive coping distraction from stress, score & $3.1(1.9)$ & $2.1(1.5)$ & 0.030 \\
\hline Positive coping control, score & $4.8(2.6)$ & $5.3(2.6)$ & 0.526 \\
\hline
\end{tabular}

Values are means with standard deviation or percentages in parentheses

BDI, Beck Depression Inventory; MDD, major depressive disorder 
Table 2. Heart rate and coagulation factor values at assessed time points per study group

\begin{tabular}{|c|c|c|c|c|c|c|c|c|}
\hline \multirow[t]{2}{*}{ Time } & \multicolumn{2}{|l|}{ Heart rate (bpm) } & \multicolumn{2}{|c|}{ Fibrinogen $(\mathrm{mg} / \mathrm{dL})$} & \multicolumn{2}{|c|}{ von Willebrand factor $(\mathrm{mg} / \mathrm{L})$} & \multicolumn{2}{|c|}{ Log D-dimer (ng/mL) } \\
\hline & Remitted MDD & Controls & Remitted MDD & Controls & Remitted MDD & Controls & Remitted MDD & Controls \\
\hline Baseline & $80.6(2.0)$ & $79.1(2.4)$ & $126.5(3.6)$ & $108.0(5.3)$ & $11.9(0.6)$ & $11.6(1.5)$ & $1.19(0.14)$ & $1.12(0.19)$ \\
\hline Post-instruction & $81.4(1.9)$ & $82.4(2.7)$ & & & & & & \\
\hline Pre-stress & $96.6(2.3)$ & $100.1(2.3)$ & & & & & & \\
\hline Post-stress & $98.3(2.2)$ & $102.5(3.1)$ & & & & & & \\
\hline$+15 \min$ & $76.6(1.4)$ & $76.6(2.4)$ & $120.4(4.2)$ & $107.0(7.8)$ & $13.6(0.7)$ & $12.5(1.1)$ & $1.55(0.09)$ & $1.57(0.17)$ \\
\hline$+30 \min$ & $74.2(1.6)$ & $75.3(2.1)$ & & & & & & \\
\hline$+45 \min$ & $75.4(1.6)$ & $73.0(2.1)$ & $119.1(4.1)$ & $106.7(6.6)$ & $13.7(0.7)$ & $12.6(0.8)$ & $1.35(0.09)$ & $1.39(0.16)$ \\
\hline
\end{tabular}

Values are means (original values) with standard error of the mean in parentheses. There was no significant group-by-time interaction for any biological measure. MDD, major depressive disorder 
Table 3. Relations between remitted MDD, negative coping, positive coping total and stress-induced coagulation activation over time

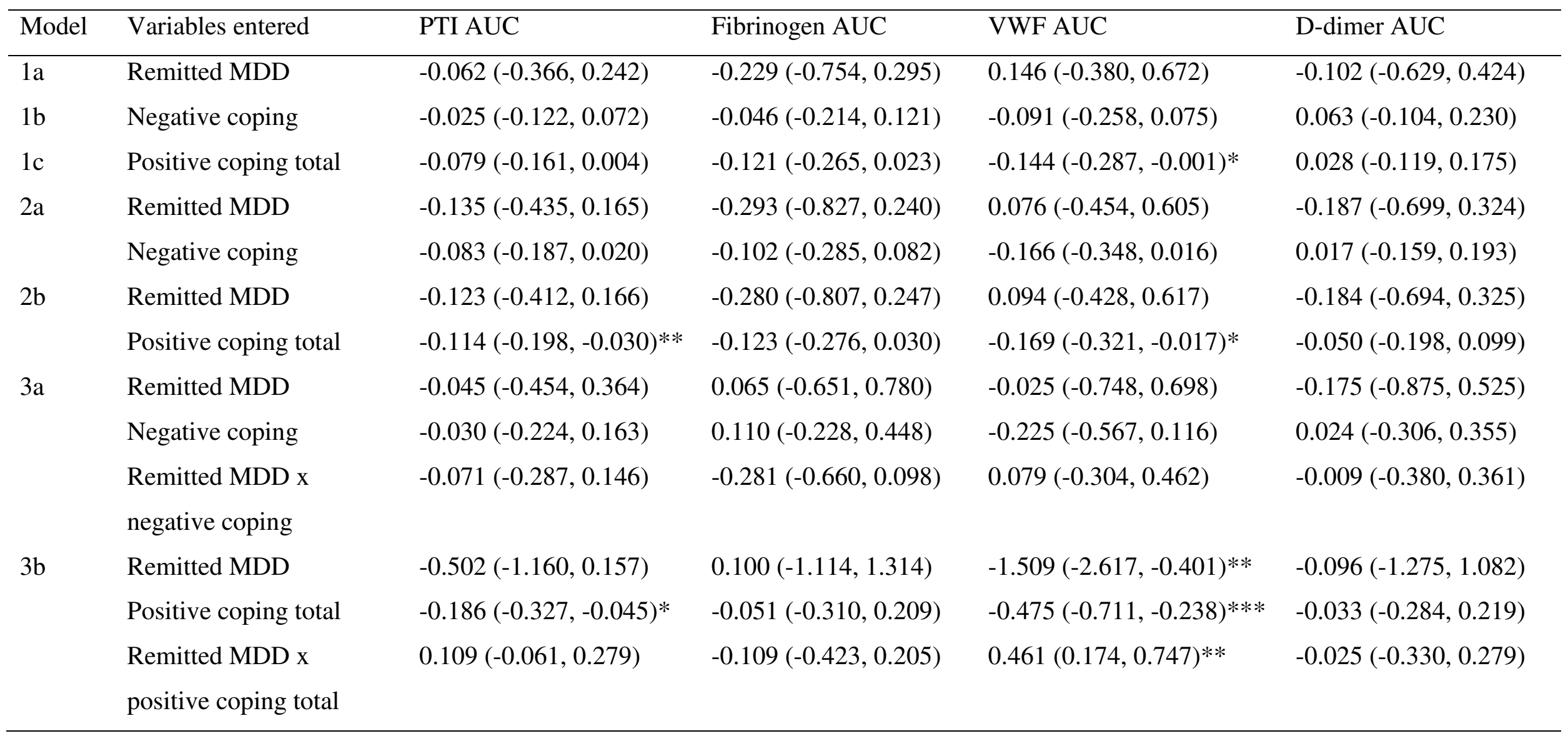

Values are unstandardized beta-coefficients (B) of z-scores with 95\% confidence interval. Model 1 shows zero-order correlations. Models 2 and 3 were both adjusted for age, sex, body mass index and smoking. Significance level: $* \mathrm{p}<0.05, * * \mathrm{P}<0.01, * * * \mathrm{P}<0.001$.

AUC, area under the curve; MDD, major depressive disorder; PTI, prothrombotic index; VWF, von Willebrand factor 
Table 4. Associations of stress-induced coagulation activation with subscales of positive coping

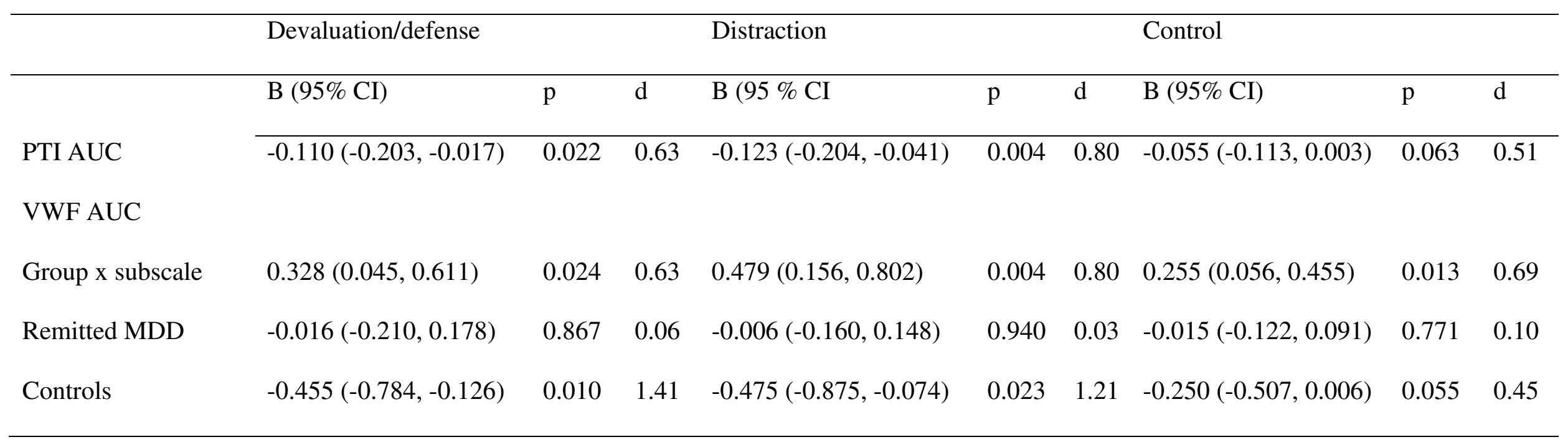

All analyses were adjusted for age, sex, body mass index and smoking.

AUC, area under the curve; MDD, major depressive disorder; PTI, prothrombotic index; VWF, von Willebrand Factor 